\title{
SANS STUDIES OF LIQUID CRYSTALLINE MICROEMULSION GELS
}

\author{
J.O. RÄDLER ${ }^{1}$, S. RADIMAN ${ }^{1}$, A. DE VALLERA ${ }^{1}$ and C. TOPRAKCIOGLU ${ }^{1,2}$ \\ ${ }^{1}$ PCS, Cavendish Laboratory, Department of Physics, University of Cambridge, Madingley Road. \\ Cambridge CB3 OHE, UK \\ ${ }^{2} A F R C$ Institute of Food Research, Colney Lane, Norwich NR4 7UA, UK
}

\begin{abstract}
We have investigated by small-angle neutron scattering (SANS) the ternary system containing water, alkane and the surfactant mixture benzyltetradecyldimethylammonium chloride and tetradecyltrimethylammonium bromide, which we have found to form a cubic liquid crystalline phase. A contrast variation experiment with equal volume fractions of water and oil showed three Bragg reflections varying in agreement with theoretically calculated scattering amplitudes for an infinite periodic minimal surface (IPMS) with cubic symmetry. We have, in addition, studied the "ringing" gel phases of the system water/octane/didodecyldimethylammonium bromide by SANS and electron microscopy.
\end{abstract}

\section{Introduction}

Microemulsions are stable mixtures of water, oil and amphiphile which normally exist in the form of transparent isotropic liquids of low viscosity. An important distinction between different topologies in microemulsion structure is whether or not they are bicontinuous [1-6]. In 1976 Scriven [1] proposed a structure based on the concept of bicontinuous infinite periodic minimal surfaces (IPMS) as an alternative to disconnected droplets. While a number of ternary systems are known to form essentially random bicontinuous phases with little or no longrange order [5], it has been much more difficult to find ternary systems exhibiting IPMS structure. In this paper we report small-angle neutron scattering (SANS) results for the system containing water, octane, and the surfactant mixture benzyldimethyl tetradecylammonium chloride (BDTAC)-tetradecyltrimethylammonium bromide (TTAB) which we have found to form a cubic liquid crystalline gel whose structure appears to conform to that of an IPMS of simple cubic symmetry as the volume fraction $\phi$ of the paraffinic phase approaches 0.5 . We also report SANS and electron microscopy (EM) measurements on the gels formed by the system water/didodecyldimethylammonium bromide (DDAB)/octane.

\section{Results and discussion}

The samples were prepared by thorough mixing of the components at $70-80^{\circ} \mathrm{C}$ for several hours and were then cooled to room temperature. They were typically subjected to several cycles of heating and quenching prior to SANS measurements, which were carried out on D17 at the ILL, Grenoble. The samples were thermostatted at $20 \pm 0.2^{\circ} \mathrm{C}$ during the SANS measurements.

\subsection{The system water/octane/BDTAC-TTAB} $(3: 1)$

We have measured the diffraction spectra of this system in two different experiments. In the first one we varied the volume fraction $\phi$ of the paraffinic phase of the system at constant water to surfactant weight ratio. Secondly, we performed a contrast experiment with a liquid crystalline gel at equal volume fractions of water and oil-like phase using unlabelled surfactant, halfdeuterated water, and changing the $\mathrm{D} / \mathrm{H}$ ratio of the octane. Fig. 1 shows the scattering spectrum obtained at $\phi=0.5$ with $100 \%$ D-octane and $50 \% \mathrm{D}_{2} \mathrm{O}$ in the aqueous phase. At least three peaks can be seen, with the higher order peaks having ratios of $\sqrt{2}$ and $\sqrt{3}$ in their $Q$-values relative to the first one. This indicates simple cubic (s.c.) or body centered cubic (b.c.c.) struc- 


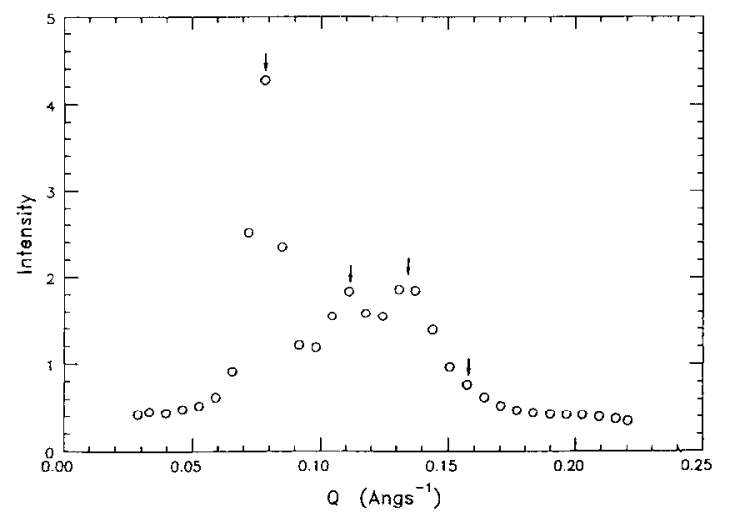

Fig. 1. Neutron scattering spectra obtained from a cubic liquid crystalline phase at $\phi=\phi_{\mathrm{w}}=0.5$. The octane is fully deuterated, while the aqueous phase contains $50 \% \mathrm{D}_{2} \mathrm{O}$. The composition (by weight) of the sample is: water $56.31 \%$, octane $14.04 \%$, BDTAC/TTAB $29.64 \%$. The arrows denote the $Q$-values corresponding to ratios of $\sqrt{2}, \sqrt{3}$ and $\sqrt{4}$ relative to the first peak.

ture with a lattice constant of $82 \AA$ (s.c.) or $115 \AA$ (b.c.c.). At $\phi$-values less than 0.5 the higher order peaks disappear, and only one peak can be observed.

\subsubsection{Variation of volume fraction}

The interfacial area $A$ of a system consisting of disconnected droplets arranged in cubic symmetry can be shown to vary with $\phi^{2 / 3}$, where $\phi$ is the volume fraction of the internal phase. If $\xi$ is the lattice constant, we can define a dimensionless area $A^{*}$, where $A^{*}(\phi)=A(\phi) / \xi^{2}$. The corresponding expressions for simple cubic and body centered cubic symmetries are:

$$
\begin{aligned}
& A^{*}(\phi)=K \phi^{2 / 3}, \text { where } \\
& K= \begin{cases}4 \pi\left(\frac{3}{4 \pi}\right)^{2 / 3} & \text { (s.c.) } \\
8 \pi\left(\frac{3}{8 \pi}\right)^{2 / 3} & \text { (b.c.c.) } .\end{cases}
\end{aligned}
$$

It can also be shown that

$A^{*}(\phi)=c_{\mathrm{s}} a_{\mathrm{s}} \xi$,

where $c_{\mathrm{s}}$ denotes the concentration of surfactant (molecules per unit volume), and $a_{\mathrm{s}}$ is the area per surfactant molecule at the interface. The lattice constant $\xi$ is related to the scattering vector position $Q_{\max }$ of a Bragg reflection:

$\xi=\frac{2 \pi}{Q_{\max }} \sqrt{h^{2}+k^{2}+l^{2}}$.

Combining (1), (2) and (3) we obtain, for a disconnected droplet system:

$\frac{c_{\mathrm{s}}}{Q_{\max }}=\frac{K \phi^{2 / 3}}{2 \pi a_{\mathrm{s}} \sqrt{h^{2}+k^{2}+l^{2}}}$.

For a bicontinuous system of water and oil separated by a surfactant interface of constant mean curvature (CMC) Anderson [7] has shown that

$A^{*}(\phi)=A^{*}\left(\phi_{\mathrm{o}}\right)-\frac{1}{c}\left(\phi-\phi_{\mathrm{o}}\right)^{2}$.

Combining (2), (3) and (5) we obtain for an IPMS structure:

$$
\begin{aligned}
\frac{c_{\mathrm{s}}}{Q_{\max }}= & \frac{1}{2 \pi a_{\mathrm{s}} \sqrt{h^{2}+k^{2}+l^{2}}} \\
& \times\left[A^{*}\left(\phi_{\mathrm{o}}\right)-\frac{1}{c}\left(\phi-\phi_{\mathrm{o}}\right)^{2}\right],
\end{aligned}
$$

where $\phi_{0}$ is the volume fraction at which the mean curvature is zero and $A^{*}\left(\phi_{\mathrm{o}}\right)$ and $c$ are constants characteristic of a particular IPMS (for the Schwarz-P surface $\phi_{\mathrm{o}}=0.5, A^{*}\left(\phi_{\mathrm{o}}\right)=2.345$, $c=0.2117$ ).

In view of the s.c. or b.c.c. indexing of our system we have considered two IPMS candidates corresponding to these symmetries: the Schwarz$P$ surface (s.c.) and the I-WP surface (b.c.c.) [7].

Fig. 2 shows a plot of $c_{\mathrm{s}} / Q_{\max }$ against $\phi_{\mathrm{w}}$ (where $\phi_{\mathrm{w}}=1-\phi$ ). As $\phi$ is increased, there is a sudden change in viscosity around $\phi=0.4\left(\phi_{\mathrm{w}}=\right.$ 0.6 ) and the system turns into a rigid gel. This is borne out by the results in fig. 2 which clearly show two distinct regimes suggesting a transition from disconnected droplets to a CMC structure. A reasonable fit is obtained for both regimes, but it is not possible to distinguish between the Schwarz-P and I-WP models, although the former appears more likely (see next section). The value of $a_{\mathrm{s}}$ extracted from the fits is modeldependent. In the $\mathrm{CMC}$ regime values of (62 \pm 4) $\AA$ and $(65 \pm 4) \AA$ are obtained for the Schwarz-P and I-WP models, respectively. The droplet regime yields values of $(77 \pm 5) \AA$ and 


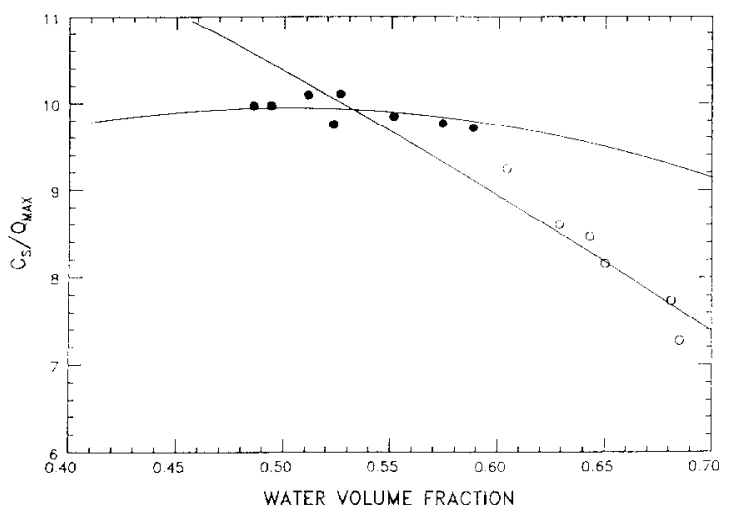

Fig. 2. Plot of $c / Q_{\max }$ against the water volume fraction $\phi_{w}$ for the system water/octane/BDTAC-TTAB (3:1) $c_{s}$ has the units of $\mathrm{mol} \mathrm{I}^{-1}$. The solid circles correspond to rigid gels, while the open circles denote liquid samples. The curves represent least squares fits to the two sets of points using the IPMS Schwarz-P model (eq. (6)) for the solid circles and the disconnected droplet model (eq. (4)) for the open ones. The only free parameter is $a$, in each case.

(68 \pm 5$) \AA$ for the s.c. and b.c.c. symmetries, respectively.

\subsubsection{Contrast variation}

The contrast experiment was carried out at $\phi=0.5$. The water phase was deuterated $50 \%$ for all measurements, while the $\mathrm{D} / \mathrm{H}$ ratio of the oil was varied. The scattering intensity can be expressed as:

$$
\begin{aligned}
I \sim & {\left[\left(\rho_{\mathrm{w}}-\rho_{\mathrm{s}}\right) \phi_{\mathrm{w}} A_{\mathrm{w}}+\left(\rho_{\mathrm{h}}-\rho_{\mathrm{s}}\right) \phi_{\mathrm{h}} A_{\mathrm{h}}\right.} \\
& \left.+\left(\rho_{\mathrm{o}}-\rho_{\mathrm{s}}\right) \phi_{\mathrm{o}} A_{\mathrm{o}}\right]^{2},
\end{aligned}
$$

where $\rho, \phi$ and $A$ represent the scattering length densities, volume fractions and scattering amplitudes of the water (w), surfactant head group (h), surfactant tail (s), and oil (o), respectively. Since $\phi_{\mathrm{h}}$ is small, we neglect the scattering from the surfactant heads. It is then easy to check that $\sqrt{I}$ is a linear superposition of two scattering amplitudes which can be determined by contrast variation (i.e. by changing $\rho_{\mathrm{o}}$ ). Anderson [7] has calculated the scattering amplitudes arising from different IPMS topologies, and our results are compared to the Schwarz-P and I-WP models in table I. As the extent of deuteration of the oil is changed from $100 \%$ to zero, the neutron scattering spectra (not shown) indicate that the second
Table I

Comparison of $A_{w}$ values extracted from the contrast variation experiment with theoretically computed values for IPMS structures.

\begin{tabular}{llcl}
\hline & Experiment & Schwarz-P $^{\text {at }}$ & I-WP $^{\text {a) }}$ \\
\hline 1st peak & -1 & -1 & -1 \\
2nd peak & -0.005 & 0.000001 & -0.194 \\
3rd peak & 0.124 & 0.362 & 0.168 \\
\hline
\end{tabular}

"Calculated from ref. [7]. All amplitudes have been normalized to the amplitude of the first peak.

peak in fig. 2 vanishes while the third peak persists. This behaviour is only consistent with the Schwarz-P model, for it follows from eq. (7) that with non-deuterated oil (i.e., $\rho_{\mathrm{o}}=\rho_{\mathrm{s}}$ ) a vanishing intensity for the second peak, implies a negligibly small $A_{\mathrm{w}}$ for that peak, since $\rho_{\mathrm{w}}-\rho_{\mathrm{s}}$ is always positive (and constant) throughout the contrast experiment. We therefore rule out the I-WP structure, which has a non-zero $A_{\mathrm{w}}$ value, larger in magnitude for the second peak than for the third peak (see table 1). Thus, the Schwarz-P structure appears to be in better agreement with the data. It should be noted that a precise comparison of the absolute values of the experimentally determined $A_{\mathrm{w}}$ 's to theory is difficult due to the Debye-Waller factor and the significant overlap between the peaks.

\subsection{The system water/octane $D D A B$}

In this system a water dilution was performed at constant surfactant to oil ratio ( $/ \mathrm{o}=3.15$ ). SANS measurements were performed using $\mathrm{D}_{2} \mathrm{O}$ and unlabelled oil and surfactant. At high $\phi_{\mathrm{w}}$ the gels showed hexagonal symmetry, while at lower values of $\phi_{w}$ there seems to be a transition to a cubic arrangement which is accompanicd by the formation of clear, tough, "ringing" gels, (i.e. these gels "ring" at a characteristic acoustic frequency when tapped). Typical SANS results are shown in fig. 3 . We have also examined the structure of these systems using freeze-fracture EM which reveals close-packed globular objects in the cubic regime (not shown). On increasing $\phi_{\mathrm{w}}$, the system appears to favour a cellular-type of arrangement. The nature of the "ringing" is not well understood. 


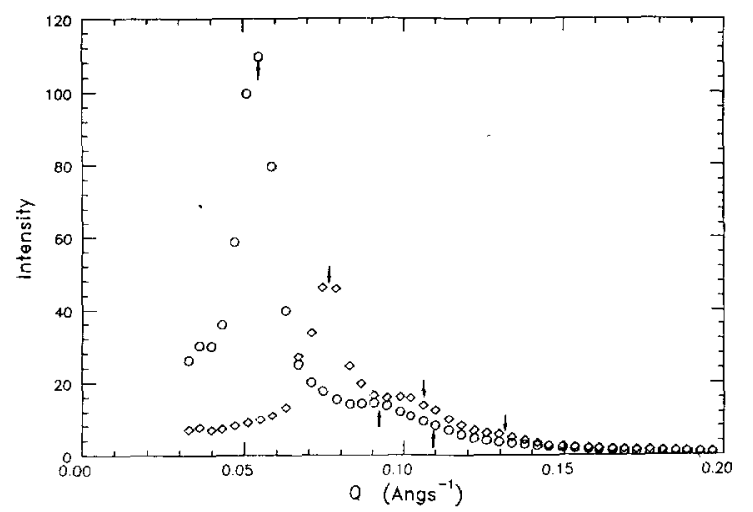

Fig. 3. Neutron scattering spectra for the system water/ octane/DDAB at two different compositions (by weight): (a) $\mathrm{D}_{2} \mathrm{O} 44.93 \%$, octane $13.27 \%$, DD $\wedge \mathrm{B} 41.80 \%$; (b) $\mathrm{D}_{2} \mathrm{O}$ $63.14 \%$, octane $8.88 \%$, DDAB $27.98 \%$. The peaks appear to index to a b.c.c. structure in (a) $\diamond$ and a hexagonal arrangement in (b) $O$, with spacings of $82 \AA$ and $116 \AA$, respectively. The arrows indicate the peak positions relative to the first peak in each case (a) $1: \sqrt{2}: \sqrt{3}$ and (b) $1: \sqrt{3}: \sqrt{4}$.

\section{Conclusions}

We have shown that the diffraction patterns of certain cubic liquid crystalline microemulsions are consistent with the concept of IPMS structure, while other systems prefer a disconnected droplet structure with cubic packing. The preference of a particular structure is related to the curvature and bending constant of the oil-water interface. It is possible that IPMS structures are a general feature of ternary systems characterized by zero mean curvature, and a relatively high interfacial bending energy.

\section{References}

[1] L.E. Scriven, Nature 263 (1976) 123.

[2] 广. Talmon and S. Prager, J. Chem. Phys. 69 (1978) 2984.

[3] P.G. de Gennes and C. Taupin, J. Phys. Chem. 86 (1982) 2294.

[4] J. Jouffroy, P. Levison and P.G. de Gennes, J. Physique 43 (1982) 1241.

[5] L. Auvray, J.P. Cotton, R. Ober and C. Taupin, J. de Physique 45 (1984) 913.

[6] J. Tabony, Nature 319 (1986) 400.

[7] D.M. Anderson, PhD Thesis, University of Minnesota, USA (1986). 